\title{
A NOTE ON THE EQUIVALENCE OF THE PARITY OF CLASS NUMBERS AND THE SIGNATURE RANKS OF UNITS IN CYCLOTOMIC FIELDS
}

\author{
DAVID S. DUMMIT ৫
}

\section{Dedicated to the memory of my teacher Kenkichi Iwasawa}

\begin{abstract}
We collect some statements regarding equivalence of the parities of various class numbers and signature ranks of units in prime power cyclotomic fields. We correct some misstatements in the literature regarding these parities by providing an example of a prime cyclotomic field where the signature rank of the units and the signature rank of the circular units are not equal.
\end{abstract}

\section{$\S 1$. Introduction}

Let $p$ be a prime and $n \geqslant 1$ a fixed integer (with $n \geqslant 2$ if $p=2$ ). Let $\zeta_{p^{n}}$ denote a primitive $p^{n}$ th root of unity, $K=\mathbb{Q}\left(\zeta_{p^{n}}\right)$ the corresponding cyclotomic field of $p^{n}$ th roots of unity, and $K^{+}=\mathbb{Q}\left(\zeta_{p^{n}}+\zeta_{p^{n}}^{-1}\right)$ the maximal totally real subfield of $K$.

Denote by $\mathrm{Cl}(K)$ the class group of $K$, by $\mathrm{Cl}\left(K^{+}\right)$the class group of $K^{+}$, and by $\mathrm{Cl}^{\text {st }}\left(K^{+}\right)$the strict (or narrow) class group of $K^{+}$.

Let $E$ denote the group of real units of $K$, that is, the units of the maximal real subfield $K^{+}$(the group of units of $K$ is then $\left\langle\zeta_{p^{n}}\right\rangle \times E$ ), and let $E^{+}$ denote the totally positive units of $K^{+}$(or, by abuse, of $K$ ).

Let $C$ denote the subgroup of circular (or cyclotomic) units of $E$ (see [26, Lemma 8.1]), whose finite index in $E$ is the class number $\left|\mathrm{Cl}\left(K^{+}\right)\right|[26$, Theorem 8.2]. Let $C^{+}$denote the subgroup of totally positive circular units (so $C^{+}=C \cap E^{+}$).

The Galois group $\operatorname{Gal}\left(K / K^{+}\right)$is generated by complex conjugation, which, following Iwasawa, will be denoted by $J$. Let $\mathrm{Cl}^{-}(K)$, the minus part of the class group, denote the kernel of $1+J$ acting on $\mathrm{Cl}(K)$. Similarly, let $\mathrm{Cl}^{+}(K)$ denote the kernel of $1-J$ acting on $\mathrm{Cl}(K)$. By class field theory, the class number of $K^{+},\left|\mathrm{Cl}\left(K^{+}\right)\right|$, divides the class number of $K$ and the norm map from $\mathrm{Cl}(K)$ to $\mathrm{Cl}\left(K^{+}\right)$is surjective, with kernel $\mathrm{Cl}^{-}(K)$, 
so $|\mathrm{Cl}(K)|=\left|\mathrm{Cl}^{-}(K)\right|\left|\mathrm{Cl}\left(K^{+}\right)\right|$. The factor $\left|\mathrm{Cl}^{-}(K)\right|$ is called the relative class number of $K$. (Warning: the group $\mathrm{Cl}\left(K^{+}\right)$embeds into $\mathrm{Cl}^{+}(K)$, but $\mathrm{Cl}^{+}(K)$ may be strictly larger. Classical terminology refers to $\left|\mathrm{Cl}\left(K^{+}\right)\right|$(and not $\left.\left|\mathrm{Cl}^{+}(K)\right|\right)$ as the "plus part" of the class number of $K$ (often as " $h^{+}$"), so to avoid confusion we shall avoid this terminology.)

Equivalencies for the parity of the orders of the various class groups and relations with signature ranks are known and due to various authors, some beginning as far back as the late 1800's with Kummer and Weber, with the first systematic study perhaps due to Hasse [13]. These equivalencies are summarized in the following proposition. For the convenience of the reader, concise proofs for these equivalencies are given later.

Proposition 1. With $K=\mathbb{Q}\left(\zeta_{p^{n}}\right), K^{+}=\mathbb{Q}\left(\zeta_{p^{n}}+\zeta_{p^{n}}^{-1}\right)$, and other notation as above, the following statements are equivalent:

(1) the class number of $K,|\mathrm{Cl}(K)|$, is odd.

(2) the relative class number of $K,\left|\mathrm{Cl}^{-}(K)\right|$, is odd.

(3) the order of $\mathrm{Cl}^{+}(K),\left|\mathrm{Cl}^{+}(K)\right|$, is odd.

(4) the strict class number of $K^{+},\left|\mathrm{Cl}^{\mathrm{st}}\left(K^{+}\right)\right|$, is odd.

(5) One of the following equivalent conditions (a1)-(a3), together with one of the following equivalent conditions (b1)-(b3), holds:

(a1) the class number of $K^{+},\left|\mathrm{Cl}\left(K^{+}\right)\right|$, is odd,

(a2) the index $[E: C]$ is odd,

(a3) $C \cap E^{2}=C^{2}$, that is, every circular unit which is a square (in $K^{+}$) is the square of a circular unit,

and

(b1) the class number and strict class number of $K^{+}$are equal,

(b2) every totally positive unit in $E$ is a square in $E: E^{+}=E^{2}$,

(b3) there are units of $K^{+}$of every possible signature.

(6) There are circular units of $K^{+}$of every possible signature (equivalently, every totally positive circular unit is the square of a circular unit: $C^{+}=C^{2}$ ).

Remark. All the statements of the proposition are known to hold when $p=2$, a result due to Weber $[27, \mathrm{~B}, \mathrm{p} .821]$. A nice proof of this result by Iwasawa (in the form of condition (1): "If $p=2, \ldots$ the class number of $Z_{e}$ $(e \geqslant 2)$ is ... odd") can be found in [15, p. 373]. 
Remark. A number of the implications in the proposition hold for more general fields, with many of the results in the literature extending to various degrees the results in Hasse's seminal work [13]. While not exhaustive, particular attention is called to the papers by Cornacchia [1-3], Garbanati [9, 10], G. Gras and M.-N. Gras [11, 12], Oriat [20], Stevenhagen [23] and the further references they contain.

The class number of $K=\mathbb{Q}\left(\zeta_{29}\right)$ is 8 and the class number of $K^{+}=$ $\mathbb{Q}\left(\zeta_{29}+\zeta_{29}^{-1}\right)$ is 1 [26, Tables, Section 3, p. 412 and Section 4, p. 421], so for this field the equivalent conditions (a1)-(a3) in (5) are satisfied, but (1) does not hold - hence also the other statements in Proposition 1 do not hold. (It is also known that (6) does not hold by the tables of Davis [4, p. 70].) This shows that the equivalent conditions (b1)-(b3) in (5) cannot be dropped (so in particular the equivalent conditions (a1)-(a3) in (5) do not imply the conditions (b1)-(b3)).

The purpose of this Note is, in addition to collecting the equivalencies of the proposition above in one place, to show (in the following section) that the units in the maximal real subfield of the cyclotomic field of $163 \mathrm{rd}$ roots of unity realize all possible signatures but the class number of $\mathbb{Q}\left(\zeta_{163}\right)$ is even, as is the relative class number " $h_{163}^{-}$." Hence for $K=\mathbb{Q}\left(\zeta_{163}\right)$, the equivalent conditions (b1)-(b3) in (5) are satisfied, but the remaining statements in Proposition 1 are not, which shows that the equivalent conditions (a1)(a3) in (5) cannot be dropped (so in particular the equivalent conditions (b1)-(b3) in (5) do not imply the conditions (a1)-(a3)). This provides a counterexample to the assertion that the circular units can be replaced by the full group of real units in equivalence (6) of Proposition 1, an error that has appeared and propagated in the literature.

In [7] the authors state that a classical result of Kummer is that every totally positive unit of $\mathbb{Q}\left(\zeta_{p}+\zeta_{p}^{-1}\right)$ is a square whenever the class number of $\mathbb{Q}\left(\zeta_{p}\right)$ is odd (which is part of the implication (1) implies (5) above), but go on to assert that "as a result of Shimura" (for which they cite [22]) "this is now extended to every totally positive unit of $\mathbb{Q}\left(\zeta_{p}+\zeta_{p}^{-1}\right)$ is a square if and only if the class number of $\mathbb{Q}\left(\zeta_{p}\right)$ is odd."

In [8], the author makes a similar statement that "With $n$ a prime power, the totally positive units in $\mathcal{O}_{n}^{+}$[the integers of the maximal real subfield of the $n$th roots of unity] are squares of units from $\mathcal{O}_{n}^{+}$if and only if $h_{n}^{-}$[the relative class number of $\left.\mathbb{Q}\left(\zeta_{n}\right)\right]$ is odd," citing Lemma 5 and Theorem 3 in [9].

It should be noted that Shimura makes no claim as asserted, in fact stating only that the converse holds (in a more general setting of imaginary 
abelian fields of prime power conductor) under the additional assumption that the class number of $\mathbb{Q}\left(\zeta_{p}+\zeta_{p}^{-1}\right)$ is odd (indicating in a footnote that this was kindly pointed out to him by Iwasawa) [22, Proposition A.5 and following, Appendix, p. 83]. Similarly, the link between the signatures of the subgroup of circular units with the signatures of the full group of units in Lemma 5 of [9] requires the class number of $\mathbb{Q}\left(\zeta_{p}+\zeta_{p}^{-1}\right)$ to be odd.

More recently, this error appears in [17], ${ }^{1}$ where the authors assert that the "Taussky conjecture" is that "every totally positive unit of $\mathbb{Q}\left(\zeta_{q}+\zeta_{q}^{-1}\right)$ is a square" in the case that both $q$ and $p=(q-1) / 2$ are primes, stating explicitly that this is equivalent to the oddness of the class number of $\mathbb{Q}\left(\zeta_{q}\right)$ (citing [7] for the equivalence). The correct conjecture (which as noted by Stevenhagen [23] appears explicitly in print only in the Ph.D. dissertation and subsequent paper of Taussky's student Davis [4, p. 4], [5] without attribution to Taussky — but note Davis references [24]) is that "every totally positive circular unit of $\mathbb{Q}\left(\zeta_{q}+\zeta_{q}^{-1}\right)$ is the square of a circular unit when $q$ and $p=(q-1) / 2$ are both primes." The terminology "Taussky's conjecture" in [17] is apparently drawn from the discussion in their reference [8], so either [7] or [8] could be the source of the confusion regarding the correct conjecture.

\section{$\S 2$. The cyclotomic field of 163rd roots of unity}

Proposition 2. If $F=\mathbb{Q}\left(\zeta_{163}\right)$ is the cyclotomic field of 163 rd roots of unity and $F^{+}=\mathbb{Q}\left(\zeta_{163}+\zeta_{163}^{-1}\right)$ is its maximal real subfield, then

(a) the units of $\mathrm{F}^{+}$have all possible signatures (that is, every totally positive unit of $F^{+}$is the square of a unit in $F^{+}$), while the subgroup of squares of circular units of $F^{+}$have index 4 in the group of totally positive circular units of $F^{+}$;

(b) the class number and strict class number of $F^{+}$are equal and divisible by 4 (with both equal to 4 under the generalized Riemann hypothesis $(G R H))$, the power of 2 in the relative class number of $F$ is 4 and the class number of $F$ is divisible by 16 (with precise 2-power divisor equal to 16 under the GRH).

In particular, every totally positive unit of $\mathrm{F}^{+}$a square does not imply that the class number (nor, equivalently, the relative class number) of $F$ is odd.

\footnotetext{
${ }^{1}$ There is also a gap in the proof of Theorem 3.3 in this paper: the " $10(r+1)$ " in the proof of Lemma 3.2 should be $10 r+11$, which need not be even.
} 
Proof. The tables of Davis [4, p. 71] show that the rank of the $81 \times 81$ matrix of signatures of the circular units in $F^{+}$is 79 , that is, $\left[C: C^{+}\right]=2^{79}$ and $\left[C: C^{2}\right]=2^{81}$, which gives the second statement in (a). The relative class number of $F$ given in [26, Tables, Section 3, p. 415] is $2^{2} \cdot 181 \cdot 23167$. $365473 \cdot 441845817162679$.

Under the GRH the class number of $F^{+}$is 4 by [25] (see also [21], whose tables are reproduced in [26, Tables, Section 4, p. 420]).

The field $F^{+}$is a cyclic extension of degree 81 over $\mathbb{Q}$ with cyclic cubic subfield $k^{+}=\mathbb{Q}(\alpha)$ where $\alpha=\operatorname{Tr}_{F^{+} / k}\left(\zeta_{163}+\zeta_{163}^{-1}\right)$, whose minimal polynomial over $\mathbb{Q}$ is $x^{3}+x^{2}-54 x-169$. The class number of $k^{+}$is 4 and $\epsilon_{1}=\alpha+4, \epsilon_{2}=\alpha^{2}-4 \alpha-34$ are fundamental units for $k^{+}$([18, 3.3.26569.1], but note the database uses $-\alpha$ as generator).

Since $F^{+} / k^{+}$is totally ramified, it follows that the class number of $F^{+}$ is divisible by 4 (and equal to 4 under the GRH, as noted above). Then the class number of $F$, which is the product of the class number of $F^{+}$with the relative class number of $F$, is divisible by 16 (with precise 2-power divisor equal to 16 under the GRH).

It remains to show that the units of $F^{+}$have all possible signatures, as this also shows the class number and strict class number of $F^{+}$are the same.

The units of $F^{+}$contain the subgroup $\left\langle\epsilon_{1}, \epsilon_{2}, C\right\rangle$ generated by the units of $k^{+}$together with the circular units of $F^{+}$. Adding the signatures of $\epsilon_{1}$ and $\epsilon_{2}$ as elements of $F^{+}$(which are easily computed since $\alpha$ is a trace) to the signature matrix for $C$ computed as in [4] produces a matrix of full rank 81 , so the full group of units of $F^{+}$also has maximal signature rank, completing the proof.

Remark. If the class number of $F^{+}=\mathbb{Q}\left(\zeta_{163}+\zeta_{163}^{-1}\right)$ is indeed equal to 4 as expected, then the index of the circular units in the units of $F^{+}$is 4 . Since the computation of the rank of the group of signatures shows $C$ has index 4 in $\left\langle\epsilon_{1}, \epsilon_{2}, C\right\rangle$, it would follow that $\left\langle\epsilon_{1}, \epsilon_{2}, C\right\rangle$ is the full group of units of $F^{+}$.

Remark. The cyclic subfield $k=k^{+}(\sqrt{-163})$ of degree 6 contained in $F$ has class group $\mathrm{Cl}(k)$ isomorphic to $(\mathbb{Z} / 2 \mathbb{Z})^{4}[18,6.0 .115063617043 .1]$. The class group of $k^{+}$is isomorphic to $(\mathbb{Z} / 2 \mathbb{Z})^{2}$ with the cyclic group $\operatorname{Gal}\left(k^{+} / \mathbb{Q}\right)$ of order 3 acting by its unique irreducible 2-dimensional representation over $\mathbb{F}_{2}$ (the finite field of order 2). Also, $\mathrm{Cl}(k) / \mathrm{Cl}^{-}(k) \simeq \mathrm{Cl}(k)^{1+J}$, which by class field theory is isomorphic to $\mathrm{Cl}\left(k^{+}\right) \simeq(\mathbb{Z} / 2 \mathbb{Z})^{2}$. It follows that $\mathrm{Cl}(k)^{-}$(which is the same as $\mathrm{Cl}(k)^{+}$since $\mathrm{Cl}(k)$ has exponent 2$)$ is isomorphic to $(\mathbb{Z} / 2 \mathbb{Z})^{2}$. 
This implies that $\mathrm{Cl}(k)$ is isomorphic as a Galois module to the direct sum of two copies of the (unique) irreducible 2-dimensional representation of the cyclic group $\operatorname{Gal}\left(k^{+} / \mathbb{Q}\right)$ of order 3 over $\mathbb{F}_{2}$, where $J$ acts by interchanging the two copies. Then composing the Hilbert class field of $k$ with $F=\mathbb{Q}\left(\zeta_{163}\right)$ shows (under the assumption of the GRH) that the Sylow 2-subgroup of $\mathrm{Cl}(F)$ is isomorphic as a Galois module to the direct sum of two copies of the (unique) irreducible 2-dimensional representation of the cyclic group $\operatorname{Gal}\left(F^{+} / \mathbb{Q}\right)$ of order 81 over $\mathbb{F}_{2}$, where $J$ acts by interchanging the two copies.

\section{$\S 3$. Proofs of the parity equivalences}

Before giving some concise proofs for the equivalencies in Proposition 1 we state a variant of a theorem of Iwasawa [15] that is quite useful (for example, [19, Proposition 2.1] is an immediate consequence).

Lemma. Suppose $L / F$ is any finite Galois extension of number fields such that

(i) the (not necessarily abelian) Galois group $\mathrm{Gal}(L / F)$ has order a power of 2 , and

(ii) the extension is unramified outside infinity and a single finite prime where the finite prime is totally ramified.

Then 2 divides the strict class number of $L$ if and only 2 divides the strict class number of $F$.

Proof. Note first it suffices to prove the result when $[L: F]=2$. Composing the strict Hilbert class field of $F$ with $L$ gives an extension of the same degree over $L$ that is unramified at finite primes, so if 2 divides the strict class number of $F$ then 2 divides the strict class number of $L$. Conversely, the strict Hilbert class field $H^{\text {st }}$ of $L$ is Galois over $F$, as is the subfield, $H^{\prime}$, fixed by $2 \mathrm{Gal}\left(H^{\text {st }} / L\right)$, and $H^{\prime}$ is an elementary abelian 2-extension of $L$. Because 2-groups acting on 2-groups necessarily have fixed points, there is a subfield of $H^{\prime}$ which is an abelian extension of $F$ of degree 4 containing $L$ as a subfield. Taking the fixed field of the inertia group for the unique ramified finite prime in this latter extension gives a quadratic extension of $F$ unramified at all finite primes, so if 2 divides the strict class number of $L$ then 2 divides the strict class number of $F$.

Proof of Proposition 1. Equivalence of (1)-(3): [16, p. 576]. Let $S(K)$ denote the Sylow 2-subgroup of $\mathrm{Cl}(K)$, with $S^{+}(K)$ (the kernel of $1-J$ ) 
and minus part $S^{-}(K)$ (the kernel of $\left.1+J\right)$. Then $S^{+}(K) \cap S^{-}(K)$ consists of the elements in $S^{+}(K)$ on which $1+J$ acts trivially, that is, the elements of order 1 or 2 in $S^{+}(K)$. Similarly, $S^{+}(K) \cap S^{-}(K)$ consists of the elements of order 1 or 2 in $S^{-}(K)$ and it follows that $S^{+}(K)$ and $S^{-}(K)$ have the same 2-rank. In particular, $S^{+}(K)=1$ if and only if $S^{-}(K)=1$. Then $S(K) / S^{-}(K)=S(K)^{1+J} \subseteq S^{+}(K)$ shows that $S(K)$ is also trivial if $S^{+}(K)=S^{-}(K)=1$. Conversely, $S(K)=1$ trivially implies $S^{+}(K)=S^{-}(K)=1$ since $S^{+}(K)$ and $S^{-}(K)$ are subgroups of $S(K)$. Hence $C(K), C^{+}(K)$, and $C^{-}(K)$ all have the same parity.

Equivalence of (1) and (4): applying the lemma to $L=\mathbb{Q}\left(\zeta_{p^{n}}\right)$ and $F=$ $\mathbb{Q}\left(\zeta_{p^{n}}+\zeta_{p^{n}}^{-1}\right)$ shows that 2 divides the class number of $K$ (which equals the strict class number as $K$ is complex) if and only if 2 divides the strict class number of $K^{+}$.

Conditions (a1) and (a2) are equivalent since $[E: C]=\left|\mathrm{Cl}\left(K^{+}\right)\right|[26$, Theorem 8.2]. To see these are equivalent to (a3), note first that $E$ and $C$ are both isomorphic to $\mathbb{Z} / 2 \mathbb{Z} \times \mathbb{Z}^{\varphi\left(p^{n}\right) / 2-1}$ as abelian groups, so the groups $E / E^{2}$ and $C / C^{2}$ have the same order $\left(=2^{\varphi\left(p^{n}\right) / 2}\right)$. This together with the isomorphism $C E^{2} / E^{2} \simeq C / C \cap E^{2}$ implies that $\left|E / C E^{2}\right|=\left[E: E^{2}\right] /\left[C E^{2}\right.$ : $\left.E^{2}\right]=\left[C: C^{2}\right] /\left[C: C \cap E^{2}\right]=\left|C \cap E^{2} / C^{2}\right|$. Hence $C \cap E^{2}=C^{2}$ if and only if $E=C E^{2}$. If $\bar{E}$ denotes the finite abelian group $E / C$, then $E=C E^{2}$ if and only if $\bar{E}=\bar{E}^{2}$, which happens if and only if $\bar{E}$ has odd order, that is, if and only if $[E: C]$ is odd.

There are units of $K^{+}$of every possible signature if and only if $\left[E: E^{+}\right]=$ $2^{\varphi\left(p^{n}\right) / 2}$, which is equivalent to $\left[E^{+}: E^{2}\right]=1$ since $2^{\varphi\left(p^{n}\right) / 2}=\left[E: E^{2}\right]=[E$ : $\left.E^{+}\right]\left[E^{+}: E^{2}\right]$, so conditions (b2) and (b3) are equivalent. Then $\left|\mathrm{Cl}^{\text {st }}\left(K^{+}\right)\right|=$ $\left|\mathrm{Cl}\left(K^{+}\right)\right|\left[E^{+}: E^{2}\right]$ (for additional details, see [6, Section 2]) shows both that (b1) is equivalent to (b2) and that (4) and (5) (in the version (a1) and (b2)) are equivalent.

The two statements in (6) are equivalent since $2^{\varphi\left(p^{n}\right) / 2}=\left[C: C^{2}\right]=[C$ : $\left.C^{+}\right]\left[C^{+}: C^{2}\right]$ so there are circular units of every possible signature (that is, $\left.\left[C: C^{+}\right]=2^{\varphi\left(p^{n}\right) / 2}\right)$ if and only if $\left[C^{+}: C^{2}\right]=1$. Then $C^{2} \subseteq C \cap E^{2} \subseteq$ $C \cap E^{+}=C^{+}$shows that $\left[C^{+}: C^{2}\right]=1$ if and only if both $C^{2}=C \cap E^{2}$ and $E^{+}=E^{2}$, so (6) is equivalent to (5) (in the version (a3) and (b2)).

Acknowledgments. I would like to thank Richard Foote, Hershy Kisilevsky, and Evan Dummit for helpful conversations. 


\section{REFERENCES}

[1] P. Cornacchia, Anderson's module for cyclotomic fields of prime conductor, J. Number Theory 67 (1997), 252-276.

[2] P. Cornacchia, The parity of the class number of the cyclotomic fields of prime conductor, Proc. Amer. Math. Soc. 125 (1997), 3163-3168.

[3] P. Cornacchia, The 2-ideal class groups of $\mathbb{Q}\left(\zeta_{l}\right)$, Nagoya Math. J. 162 (2001), 1-18.

[4] D. L. Davis, On the distribution of the signs of the conjugates of the cyclotomic units in the maximal real subfield of the qth cyclotomic fields, q a prime, Ph.D. dissertation, CalTech, Pasadena, California, 1969, available from http://thesis.library.caltech.ed u/9554/.

[5] D. L. Davis, Computing the number of totally positive circular units which are squares, J. Number Theory 10 (1978), 1-9.

[6] D. Dummit and J. Voight, The 2-Selmer group of a number field and heuristics for narrow class groups and signature ranks of units, Proc. Lond. Math. Soc. (3) 117 (2018), 682-726.

[7] H. Edgar, R. Mollin and B. Peterson, Class groups, totally positive units, and squares, Proc. Amer. Math. Soc. 98 (1986), 33-37.

[8] D. R. Estes, On the parity of the class number of the field of q th roots of unity, Rocky Mountain J. Math. 19 (1989), 675-682.

[9] D. Garbanati, Unit signatures, and even class numbers, and relative class numbers, J. Reine Angew. Math. 274/5 (1975), 376-384.

[10] D. Garbanati, Units with norm -1 and signatures of units, J. Reine Angew. Math. 283/4 (1976), 164-175.

[11] G. Gras, Parité du nombre de classes et unités cyclotomiques, Asterisque 24/25 (1975), 37-45.

[12] G. Gras and M.-N. Gras, Signature des unités cyclotomiques et parité du nombre de classes des extensions cycliques de $Q$ de degré premier impair, Ann. Inst. Fourier, Grenoble 25 (1975), 1-22.

[13] H. Hasse, Über die Klassenzahl abelscher Zahlkörper, Akademie-Verlag, Berlin, 1952.

[14] I. Hughes and R. Mollin, Totally positive units and squares, Proc. Amer. Math. Soc. 87(4) (1983), 613-616.

[15] K. Iwasawa, "A note on class numbers of algebraic number fields", in Kenkichi Iwasawa Collected Papers I, (eds. I. Satake et al.) Springer, Tokyo, 2001, 372-373. (original work published 1956).

[16] K. Iwasawa, "A note on ideal class groups", in Kenkichi Iwasawa Collected Papers II, (eds. I. Satake et al.) Springer, Tokyo, 2001, 239-247. (original work published 1966).

[17] M.-H. Kim and S.-G. Lim, Square classes of totally positive units, J. Number Theory 125 (2007), 1-6.

[18] The LMFDB Collaboration: The L-functions and Modular Forms Database, http:// www.lmfdb.org, 2013 [Online; accessed November 2017].

[19] O. Neumann, "On Maximal p-Extensions, Class Numbers and Unit Signatures", Journées Arithmétiques de Caen (Univ. Caen, Caen, 1976), Asterisque 41-42, Soc. Math. France, Paris, 1977, 239-246.

[20] B. Oriat, Relation entre les 2-groupes de classes d'idéaux au sens ordinaire et restreint de certains corps de nombres, Bull. Soc. Math. France 104 (1976), 301-307.

[21] R. Schoof, Class numbers of real cyclotomic fields of prime conductor, Math. Comput. 72 (2003), 913-937. 
[22] G. Shimura, On abelian varieties with complex multiplication, Proc. Lond. Math. Soc. (3) 34 (1977), 65-86.

[23] P. Stevenhagen, Class number parity for the pth cyclotomic field, Math. Comput. 63 (1994), 773-784.

[24] O. Taussky, Unimodular integral circulants, Math. Z. 63 (1955), 286-298.

[25] F. Van der Linden, Class number computations of real abelian number fields, Math. Comput. 39 (1982), 693-707.

[26] L. Washington, Introduction to Cyclotomic Fields, 2nd ed., Springer, New York, 1997.

[27] H. Weber, Lehrbuch der Algebra, 3rd ed., Vol. II, AMS Chelsea Publishing, Providence, RI, 2000, (Reprint of 1899 second edition).

Department of Mathematics

University of Vermont

Lord House

16 Colchester Ave.

Burlington

VT 05405

$U S A$

dummit@math.uvm.edu 